\title{
Neuronavigation-guided high-dose repetitive transcranial magnetic stimulation for the treatment of depressive adolescents with suicidal ideation: a case series
}

This article was published in the following Dove Press journal:

Neuropsychiatric Disease and Treatment

\author{
Fen $\operatorname{Pan}^{1,2}$ \\ $\mathrm{Da} \mathrm{Li} \mathrm{i}^{1,2}$ \\ Xiaojing Wang ${ }^{3}$ \\ Shaojia Lu ${ }^{1,2}$ \\ Yi $X u^{1,2}$ \\ Manli Huang ${ }^{1,2}$ \\ 'Department of Mental Health, The \\ First Affiliated Hospital, College \\ of Medicine, Zhejiang University, \\ Hangzhou 310003 , People's Republic \\ of China; ${ }^{2}$ The Key Laboratory of \\ Mental Disorder's Management of \\ Zhejiang Province, Hangzhou 310003 , \\ People's Republic of China; ${ }^{3}$ School \\ of Medicine, Zhejiang University, \\ Hangzhou 310000, People's \\ Republic of China
}

\begin{abstract}
Background: A high proportion of adolescents with major depressive disorder currently do not respond to conventional treatment. Repetitive transcranial magnetic stimulation (rTMS) is a promising treatment for major depressive disorder.

Case: We report on 3 cases of adolescents with suicidal ideation receiving 7 daily $10 \mathrm{~Hz}$ left prefrontal rTMS combined with pharmacotherapy treatment over 1 week for major depressive episode. The left dorsolateral prefrontal cortex was identified using magnetic resonance imagingguided neuronavigation prior to stimulation. The suicidal ideation of these patients lessened significantly following rTMS treatment. Regarding adverse effects, symptoms of hypomania occurred in two patients since day 4, but no other side effects were found.

Conclusion: Neuronavigation-guided high-dose rTMS may be an effective and feasible treatment option for depressive adolescents with suicidal ideation. Caution over treatment-emergent mania/hypomania associated with rTMS is required.
\end{abstract}

Keywords: neuronavigation, repetitive transcranial magnetic stimulation, depressive adolescents, suicidal ideation

\section{Introduction}

Major depressive disorder (MDD), with a prevalence of $4 \%-8 \%$, is the single greatest cause of disability and morbidity in adolescents. ${ }^{1}$ Compared to adult-onset depression, adolescent-onset depression is associated with higher rates of suicide. ${ }^{2}$ Selective serotonin reuptake inhibitors and cognitive behavioral therapy are considered to be the standard treatment for severe or treatment-resistant depression. However, up to $40 \%$ of MDD adolescents currently do not respond to conventional treatment. ${ }^{3}$ Thus, a well-tolerated and effective therapeutic modality for the treatment of adolescent depression is essential. Multiple large randomized clinical trials have proven that repetitive transcranial magnetic stimulation (rTMS) was an effective clinical treatment for MDD. ${ }^{4}$ High-dose deep TMS has shown a strong, specific, and progressive antisuicide effect in adults, ${ }^{5}$ while intensive rTMS has shown a rapid antisuicide effect in a suicidal sample. ${ }^{6}$ As far as we know, there are no published studies on the use of high-dose rTMS in MDD adolescents specifically focusing on suicidal ideation. In addition, magnetic resonance imaging (MRI)-based neuronavigation is a good technique for localization owing to its characteristics of accuracy and individualization. ${ }^{7}$ The cases described below were from the preliminary experiment for a double-blind sham-controlled 
study of 1 week of neuronavigation-guided daily high-dose rTMS combined with pharmacotherapy for the treatment of depressive adolescents with suicidal ideation.

\section{Case series}

\section{Assessments and rTMS procedure}

All subjects met the Diagnostic and Statistical Manual of Mental Disorders, IV Edition (DSM-IV) criteria for current unipolar MDD which was assessed using structured clinical interviews based on the DSM-IV (SCID) by two professional psychiatrists. All patients scored $\geq 12$ on the Beck Scale for Suicide Ideation-Chinese Version (BSI-CV) ${ }^{8}$ and $\geq 20$ on the Montgomery-Asberg Depression Rating Scale (MADRS) ${ }^{9}$ at baseline. The patients were experiencing their first episode of depression and were antidepressant drug naive, or were experiencing recurrent depression and not taking antidepressants for nearly 2 weeks. Primary outcomes were measured by the BSI-CV administered at baseline, day 3, and 7 and the MADRS administered at baseline and day 7 .

The rTMS treatment was delivered by a Magstim ${ }^{\circledR}$ magnetic stimulator (The Magstim Company Ltd, Whitland, UK) with an eight-figured coil. We defined the left dorsolateral prefrontal cortex target using MRI data by an ultrasound-based navigation system, which enables the identification of anatomical landmarks $(-44,40,29)^{10}$ using an MRI reconstruction of each patient's head (BrainVoyager TMS Neuronavigator, Brain Innovation; Maastricht, the Netherlands) (Figure 1) and coregisters the actual head with an ultrasound-based coregistration device (Zebris Medical GmbH; Isny im Allgäu, Germany), to lead the rTMS coils to target the locations of interest consistently. ${ }^{11} \mathrm{We}$ applied rTMS treatment for 7 consecutive days over 1 week. Each daily rTMS session comprised 120 trains of 5 seconds duration at $10 \mathrm{~Hz}$ with intertrain intervals of 15 seconds (ie, 6,000 pulses per session). The total number of stimuli in a given day or week was slightly higher than that in the published safety guidelines, ${ }^{12}$ but lower than the parameters of a 3-day trial of high-dose left prefrontal rTMS for adult suicidal patients, which confirmed the safety of high-dose parameters. ${ }^{6}$ The stimulus intensity was $100 \%$ of the resting motor threshold (MT) based on the tolerance of the participant. The MT was defined as the lowest intensity of TMS required to induce a motor response in the contralateral resting abductor pollicis brevis muscle.

\section{Patient I}

A 16-year-old female was admitted to our hospital due to a 2-year history of depressed emotions and decreased social activity. Her symptoms got worse since half a year ago after learning that she was adopted. She took all her antidepressant

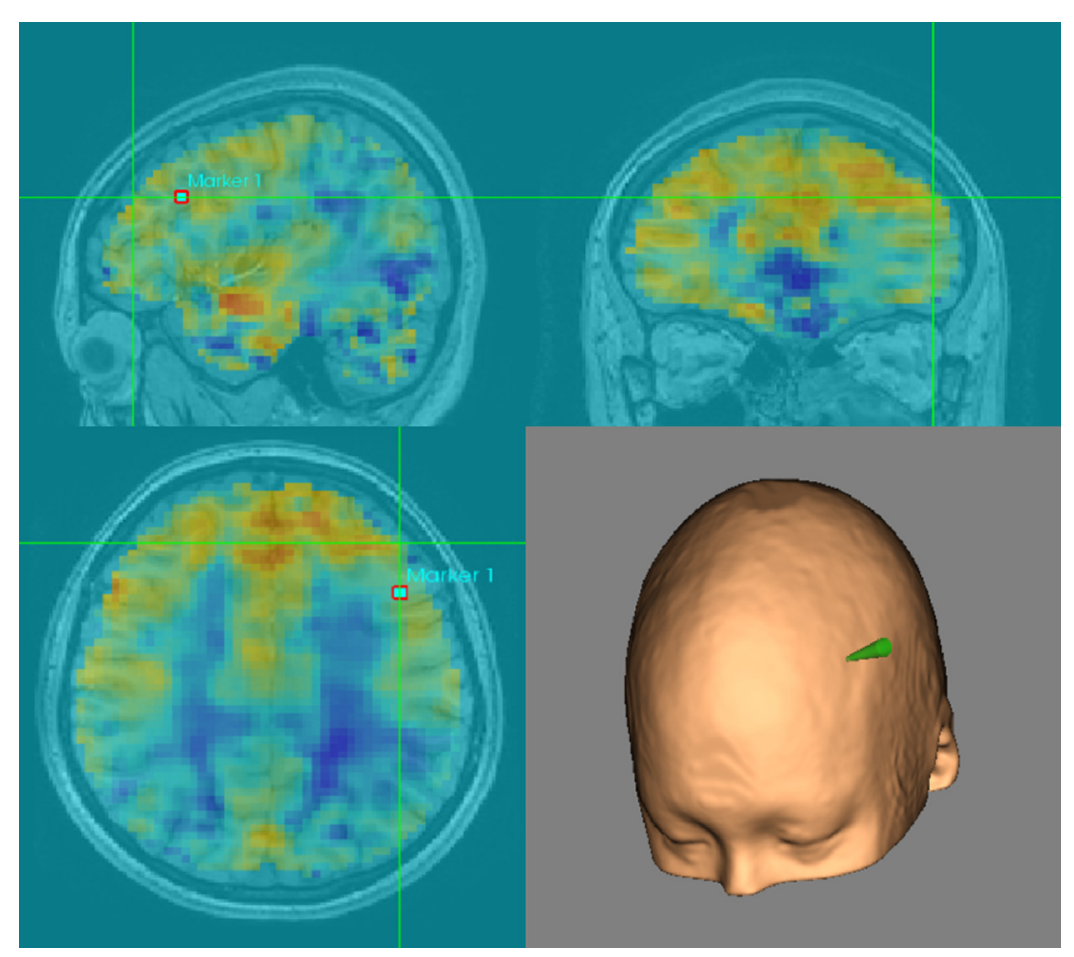

Figure I The left dorsolateral prefrontal cortex $(-44,40,29)$ was targeted using a MRI-guided neuronavigation system, and the location of the TMS coil was marked on a cap. Abbreviations: MRI, magnetic resonance imaging; TMS, transcranial magnetic stimulation. 
drugs 3 months ago and slashed her wrists 10 days ago. Fortunately, there was no serious consequence. The patient reported no family history and past history of manic episode and psychosis. There was also no alcohol or substance abuse and significant medical illness. Her medication before and during rTMS included escitalopram oxalate tablets $(10 \mathrm{mg} / \mathrm{d})$. Her baseline total scores on the BSI-CV and MADRS were 60.61 and 41, respectively. By the end of the treatment, her total scores on the BSI-CV and MADRS were 36.36 (40.01\% improvement) and 35 (14.63\% improvement), respectively (Table 1, Figure 2). She reported no subjective side effects from rTMS except for feeling sleepy after every rTMS session.

\section{Patient 2}

A 17-year-old male was admitted to our hospital due to a 6-month history of depressed emotions, anhedonia, and insomnia. Triggers to the onset of the depressive episode included domestic trivia and stress of studying. He felt that there was no point for living and had suicidal ideation. $\mathrm{He}$ felt inferior sometimes. Moreover, his memory declined and concentration dropped. As a consequence, his school performance declined, and he began to regularly miss school or leave school early. He had not received any treatment before coming to our hospital. The patient reported no family history and past history of manic episode and psychosis. There was also no alcohol or substance abuse or significant medical illness. His medication during rTMS included escitalopram oxalate tablets $(10 \mathrm{mg} / \mathrm{d})$. His baseline total scores on the BSI-CV and MADRS were 36.36 and 27, respectively. By the end of the treatment, his total scores on the BSI-CV and MADRS were 0 (100\% improvement) and 19 (29.63\% improvement), respectively (Table 1, Figure 2 ). He reported no subjective side effects from rTMS except for feeling sleepy after every rTMS session. Symptoms of hypomania

Table I Clinical tests

\begin{tabular}{c|l|l|l}
\hline & Baseline & Day 3 & Week I \\
\hline Patient I & & & \\
BSI-CV & 60.61 & 54.54 & 36.36 \\
MADRS & $4 I$ & - & 35 \\
Patient 2 & & & \\
BSI-CV & 36.36 & 30.30 & 0 \\
MADRS & 27 & - & 19 \\
Patient 3 & & & \\
BSI-CV & 72.73 & 51.52 & 18.18 \\
MADRS & 46 & - & 24 \\
\hline
\end{tabular}

Abbreviations: BSI-CV, Beck Scale for Suicide Ideation-Chinese Version; MADRS, Montgomery-Asberg Depression Rating Scale.

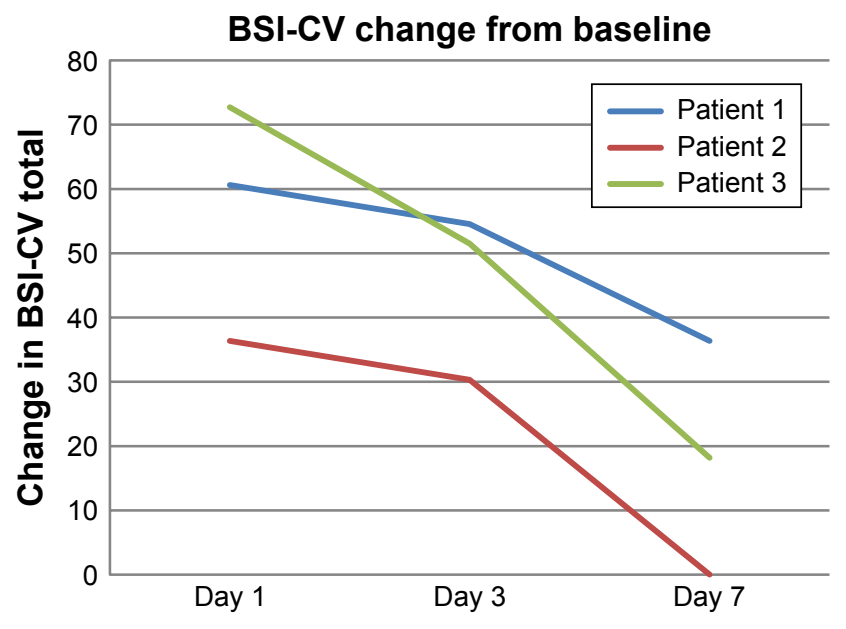

Figure 2 Results of suicide ideation assessment over the I-week treatment period Abbreviation: BSI-CV, Beck Scale for Suicide Ideation-Chinese Version.

occurred since day 4 . He became talkative and felt hopeful about the future especially in the afternoon. But he could not be diagnosed with hypomania according to the results of Hypomania Symptom Checklist- $32^{13}$ and the Mood Disorder Questionnaire. ${ }^{14} \mathrm{He}$ was discharged from the hospital after rTMS treatment because he had to go to school; thus, we lost him to follow-up.

\section{Patient 3}

A 15-year-old female was admitted to our hospital due to a 6-month history of depressed mood. She did not want to speak to her classmates, especially boys. She was in bad health for some time and often caught a cold. Then, she felt useless and reproached herself. Her symptoms got worse after getting poor scores in the final examination 2 months ago. Her memory declined and concentration dropped. Suicidal ideation appeared with her desire to jump out of the window at school. She slashed the back of her hands and wrists several times 1 week ago. Fortunately, there was no serious consequence. The patient reported no family history and past history of manic episode and psychosis. There was also no alcohol or substance abuse and significant medical illness. She took sertraline hydrochloride $(50 \mathrm{mg} / \mathrm{d})$ for 2 days and discontinued the medication for difficulty falling asleep before coming to our hospital. Her medication before and during rTMS included escitalopram oxalate tablets $(10 \mathrm{mg} / \mathrm{d})$. Her baseline total scores on the BSI-CV and MADRS were 72.72 and 46, respectively. By the end of the treatment, her total scores on the BSI-CV and MADRS were 18.18 (75\% improvement) and 24 (47.83\% improvement), respectively (Table 1, Figure 2). She reported no subjective side effects from rTMS except for feeling sleepy after every rTMS 
session. Meanwhile, symptoms of hypomania occurred since day 4 . She became talkative and had a strange sense of elation, especially in the afternoon. She was diagnosed with hypomania according to the results of Hypomania Symptom Checklist-32 and Mood Disorder Questionnaire. Thus, escitalopram oxalate tablets were discontinued and sodium valproate $(500 \mathrm{mg} / \mathrm{d})$ was introduced after 1 week of rTMS treatment. Her mood gradually became stable after changing the medication. One month later, the patient only complained of feeling a bit down. Her suicidal thoughts had disappeared and the medication remained unchanged.

\section{Informed consent}

The publication of this case series was approved by the ethics committee of the First Affiliated Hospital of the College of Medicine of Zhejiang University. Written informed consent was obtained from all participants and their parents or legal guardians for the case details to be published.

\section{Discussion}

In our cases, neuronavigation-guided high-dose rTMS was effective and safe when used as a therapeutic modality for depressive adolescents with suicidal ideation. One patient's suicidal ideation disappeared after seven sessions of rTMS. The finding was consistent with result in an adult rTMS treatment study, which demonstrated that high doses of rTMS delivered over 3 days proved to be efficacious for suicidal patients. ${ }^{6}$ The treatment outcomes in depressive symptoms of the present cases were consistent with a previous rTMS study in adolescent depression with a different localization mode and total number of stimuli. ${ }^{15}$ However, to our knowledge, neuronavigation-guided high-dose rTMS has never been studied in the treatment for suicidal, depressive adolescents. Overall, the clinically significant improvement in these cases provides evidence for the use of neuronavigation-guided high-dose rTMS to treat depression in adolescents. There were several limitations in the current report. First, the sample size was small. Second, findings could likely represent regression to the mean as suicidal ideation often improves over time with structure.

It is worthwhile to note that symptoms of hypomania occurred in two patients since day 4 . The adolescents in the present report had no history of hypomania or mania prior to rTMS treatment. Treatment-emergent mania/hypomania associated with rTMS in depressive adolescents and adults has been reported before. ${ }^{16,17}$ The episode of hypomania might be provoked by rTMS alone or in combination with the antidepressant, or possibly by the antidepressant alone. ${ }^{17}$
Another possibility is that the two patients with hypomania episode could have been misdiagnosed with MDD. The majority of adolescents with bipolar disorder experienced a depressive episode as the onset mood episode. ${ }^{18}$ This phenomenon also prompted us to set the parameters for rTMS. It seems that both high-frequency and low-frequency rTMS with light or robust stimulus parameters may contribute to the episode of hypomania. ${ }^{17}$ High-dose rTMS (6,000 stimuli per session) has not been used in adolescents before, and so further studies are needed to establish the most fit and safe stimulus parameters.

\section{Conclusion}

In summary, neuronavigation-guided high-dose rTMS can be used to rapidly reduce suicidal ideation and improve depressive symptoms. Caution over treatment-emergent mania/hypomania associated with rTMS may be required. Further well-designed, sham-controlled studies are needed to test the efficacy and safety of high-dose rTMS in depressed adolescents.

\section{Acknowledgments}

The authors thank the patients for their consent to publish this article. This work was supported by grants $2015 \mathrm{C} 03040$ from the Major Subject of Zhejiang Province, grants 81301158 and 81601182 from the National Natural Science Foundation of China, grant 2016YFC1307100 from the National Key Research and Development Program of China, and grants 2013RCB005 from the Department of Health Foundation of Zhejiang Province. The funding sources had no role in the study design; collection, analysis, and interpretation of data; writing of the manuscript; or decision to submit the paper for publication.

\section{Disclosure}

The authors report no conflicts of interest in this work.

\section{References}

1. Thapar A, Collishaw S, Pine DS, Thapar AK. Depression in adolescence. Lancet. 2012;379(9820): 1056-1067.

2. Thapar A, Collishaw S, Pine DS, Thapar AK. Depression in adolescence. Lancet. 2012;379(9820):1056-1067.

3. March J, Silva S, Petrycki S, et al. Treatment for Adolescents With Depression Study (TADS) Team. Fluoxetine, cognitive-behavioral therapy, and their combination for adolescents with depression: Treatment for Adolescents with Depression Study (TADS) randomized controlled trial. JAMA. 2004;292(7):807-820.

4. Perera T, George MS, Grammer G, Janicak PG, Pascual-Leone A, Wirecki TS. The Clinical TMS Society Consensus Review and Treatment Recommendations for TMS Therapy for Major Depressive Disorder. Brain Stimul. 2016;9(3):336-346. 
5. Rapinesi C, Kotzalidis GD, Scatena P, et al. Alcohol and suicidality: could deep transcranial magnetic stimulation (dTMS) be a possible treatment? Psychiatr Danub. 2014;26(3):281-284.

6. George MS, Raman R, Benedek DM, et al. A two-site pilot randomized 3 day trial of high dose left prefrontal repetitive transcranial magnetic stimulation (rTMS) for suicidal inpatients. Brain Stimul. 2014;7(3): 421-431.

7. Rusjan PM, Barr MS, Farzan F, et al. Optimal transcranial magnetic stimulation coil placement for targeting the dorsolateral prefrontal cortex using novel magnetic resonance image-guided neuronavigation. Hum Brain Mapp. 2010;31(11):1643-1652.

8. Beck AT, Kovacs M, Weissman A. Assessment of suicidal intention: the Scale for Suicide Ideation. J Consult Clin Psychol. 1979;47(2): 343-352.

9. Montgomery SA, Asberg M. A new depression scale designed to be sensitive to change. Br J Psychiatry. 1979;134:382-389.

10. Fox MD, Buckner RL, White MP, Greicius MD, Pascual-Leone A. Efficacy of transcranial magnetic stimulation targets for depression is related to intrinsic functional connectivity with the subgenual cingulate. Biol Psychiatry. 2012;72(7):595-603.

11. Hayasaka S, Nakamura M, Noda Y, et al. Lateralized hippocampal volume increase following high-frequency left prefrontal repetitive transcranial magnetic stimulation in patients with major depression Psychiatry Clin Neurosci. 2017;71(11):747-758.
12. Rossi S, Hallett M, Rossini PM, Pascual-Leone A. Safety of TMS Consensus Group. Safety, ethical considerations, and application guidelines for the use of transcranial magnetic stimulation in clinical practice and research. Clin Neurophysiol. 2009;120(12):2008-2039.

13. Angst J, Adolfsson R, Benazzi F, et al. The HCL-32: towards a selfassessment tool for hypomanic symptoms in outpatients. $J$ Affect Disord. 2005;88(2):217-233.

14. Hirschfeld RM, Williams JB, Spitzer RL, et al. Development and validation of a screening instrument for bipolar spectrum disorder: the Mood Disorder Questionnaire. Am J Psychiatry. 2000;157(11):1873-1875.

15. Wall CA, Croarkin PE, Maroney-Smith MJ, et al. Magnetic Resonance Imaging-Guided, Open-Label, High-Frequency Repetitive Transcranial Magnetic Stimulation for Adolescents with Major Depressive Disorder. J Child Adolesc Psychopharmacol. 2016;26(7):582-589.

16. Bloch Y, Grisaru N, Harel EV, et al. Repetitive transcranial magnetic stimulation in the treatment of depression in adolescents: an open-label study. J Ect. 2008;24(2):156-159.

17. Rachid F. Repetitive Transcranial Magnetic Stimulation and Treatmentemergent Mania and Hypomania: A Review of the Literature. J Psychiatr Pract. 2017;23(2):150-159.

18. Lewinsohn PM, Klein DN, Seeley JR. Bipolar disorders in a community sample of older adolescents: prevalence, phenomenology, comorbidity, and course. J Am Acad Child Adolesc Psychiatry. 1995;34(4): 454-463.
Neuropsychiatric Disease and Treatment

\section{Publish your work in this journal}

Neuropsychiatric Disease and Treatment is an international, peerreviewed journal of clinical therapeutics and pharmacology focusing on concise rapid reporting of clinical or pre-clinical studies on a range of neuropsychiatric and neurological disorders. This journal is indexed on PubMed Central, the 'PsycINFO' database and CAS,

\section{Dovepress}

and is the official journal of The International Neuropsychiatric Association (INA). The manuscript management system is completely online and includes a very quick and fair peer-review system, which is all easy to use. Visit http://www.dovepress.com/testimonials.php to read real quotes from published authors. 